\title{
COCCIÓN DE LADRILLOS MACIZOS EN HORNOS A FUEGO DORMIDO Y CONTINUO Y DETERMINACION DE SUS PROPIEDADES MECÁNICAS
}

\section{FIRING OF SOLID BRICKS IN INTERMITTENT AND CONTINUOUS KILNS, AND DETERMINATION OF THEIR MECHANICAL PROPERTIES}

\author{
MSc. Gustavo Guerrero Gómez * , Dr. Eng. Carlos J. Noriega Sanchez ${ }^{* *}$, Dr. Eng. \\ Nelson Afanador García*** \\ * Universidad Francisco de Paula Santander Ocaña, Facultad de Ingeniería, \\ Vía Acolsure, sede Algodonal, Ocaña, Norte de Santander, Colombia. \\ Teléfono y Fax, con indicativos internacional y nacional. \\ E-mail: gguerrerog@ufpso.edu.co. \\ * * Universidad Francisco de Paula Santander, Facultad de Ingeniería, Grupo de \\ Investigación en tecnología y desarrollo en ingeniera (GITYD). \\ Vía Acolsure, Sede el Algodonal, Ocaña, Norte de Santander, Colombia. \\ Tel. +57-7- 5690088 Ext. 221. \\ **** Universidad Francisco de Paula Santander, Facultad de Ingeniería, Grupo de \\ Investigación en Ingeniera Civil (GIIC). \\ Vía Acolsure, Sede el Algodonal, Ocaña, Norte de Santander, Colombia. \\ Tel. +57-7- 5690088 Ext. 218. \\ E-mail: nafanadorg@ufpso.edu.co.
}

Resumen: El artículo presenta la cocción de ladrillos macizos en horno natural tipo fuego dormido y en un horno continuo tipo Hoffman ,los cuales fueron fabricados en la ladrillera la Pradera, y la comparación de sus propiedades mecánicas, a las cuales se les realizaron ensayos de tasa de absorción inicial, inmersión durante 24 horas, resistencia a la compresión y módulo de rotura o flexión de acuerdo la norma técnica Colombina NTC4017 "Métodos para muestreo y ensayos de unidades de mampostería y otros productos de arcilla". Para los ensayos de resistencia a la compresión y módulo de rotura se utilizó una máquina Universal tipo Pinzuar PU-100 y el software estadístico Statgraphics.

Los resultados se compararon entre la cocción en los dos hornos y con los valores de referencia establecidos en la norma NTC 4205 unidades de mampostería de arcilla cocida, ladrillos y bloques cerámicos.

Palabras clave: Horno a fuego dormido, Horno continuo, Proceso de cocción, Máquina Universal, Propiedades mecánicas

Abstract: This article presents the firing process of solid bricks in intermittent and Hoffmann continuous kilns. The bricks were manufactured in the brickworks La Pradera, and the comparison of their mechanical properties was carried out. Also, tests of initial absorption rate, water immersion, compressive strength and modulus of rupture according to the Colombian NTC-4017 technical standard "Methods for sampling and testing of masonry units and other clay products" were performed. For the Compressive strength and modulus of rupture tests, a universal PU testing machine and the statistical software Statgraphics were used. The results of the firing process in the two kilns were compared with the reference values established in the NTC 4205 standard for fired clay masonry units, bricks and ceramic blocks.

Keywords: Intermiten kiln, Continuous kiln, firing process, Universal testing machine, mechanical properties 


\section{INTRODUCCIÓN}

En el municipio de Ocaña departamento de Norte de Santander, la fabricación de materiales cerámicos se hace de manera empírica y artesanal (Guerrero, 2017), utilizando hornos que utilizan como combustible carbón y leña (García, 2019), su combustión es incompleta (Guerrero, 2017), y fabrican 1.027.600 productos por mes tales como ladrillo, bloque y tejas (Guerrero ,2017); (L Tangarife et al., 2017).

El sector cerámico genera una cantidad significativa de empleos directos y disminuyendo los índices de pobreza (Rincón, 2016).

Los materiales cerámicos producidos son utilizados en la construcción de obras civiles, por sus bajos costos (Rozo, 2014), estos adquieren durante la cocción propiedades y características deseadas fijadas por las normas (Aranguren, 2011). Sin embargo, debido a la acción de cargas verticales u horizontales que se presentan en las construcciones se pueden generar fracturas (Asociación de Ingeniería Sísmica, AIS 2004) y si su calidad no es adecuada aumenta la vulnerabilidad sísmica (Cuellar, 2006); (J Plaza, M Núñez, 2017).

En la fabricación de materiales cerámicos se han utilizado cenizas volantes, escorias y barros (Francis ,2002), vidrios y cerámicos (Barbieri ,1999), cenizas de biomasa (Souza, 1999) y estériles de explotación minera (Skarkynska ,1999); (O Suarez at el, 2018).

Esta investigación tuvo como objetivo determinar las propiedades mecánicas de los ladrillos macizos producidos en la ladrillera la Pradera y horneados en un horno artesanal a fuego dormido y en un horno continuo, y compararlas siguiendo los lineamientos de la norma NTC 4017 métodos para muestreo y ensayos de unidades de mampostería y otros productos de arcilla (Instituto Colombiano De Normas Técnicas NTC 4017, 2005) y el cumplimiento de propiedades establecidas en la norma NTC 4205 unidades de mampostería de arcilla cocida, ladrillos y bloques cerámicos( Instituto Colombiano De Normas Técnicas NTC $4205,2000)$.

\section{METODOLOGÍA}

El estudio se realizó en dos hornos de producción de material cerámico en el municipio de Ocaña, Norte de Santander, en la primera parte de la investigación se realizó una cocción de ladrillo macizo en un horno artesanal a fuego dormido construido de ladrillo común de la ladrillera la Pradera, de sección transversal circular de diámetro interior 2.12 metros, altura 4.52 metros y espesor de pared de 0.24 metros. En esta horneada se produjeron 4.300 ladrillos, se consumieron 1.500 kg de carbón y tuvo una duración de tres días.

El proceso de preparación de la arcilla y el moldeo del ladrillo se hace de manera manual, el secado de los ladrillos se hace de forma natural a exposición directa del sol, en el proceso de cargue se colocan ladrillos de forma ordenada y en los espacios que quedan entre los ladrillos se deposita el carbón necesario para la combustión, (Ver figura 1). Al terminar de cargar el horno se sella la puerta de cargue y descargue de productos a través de una pared de ladrillos.

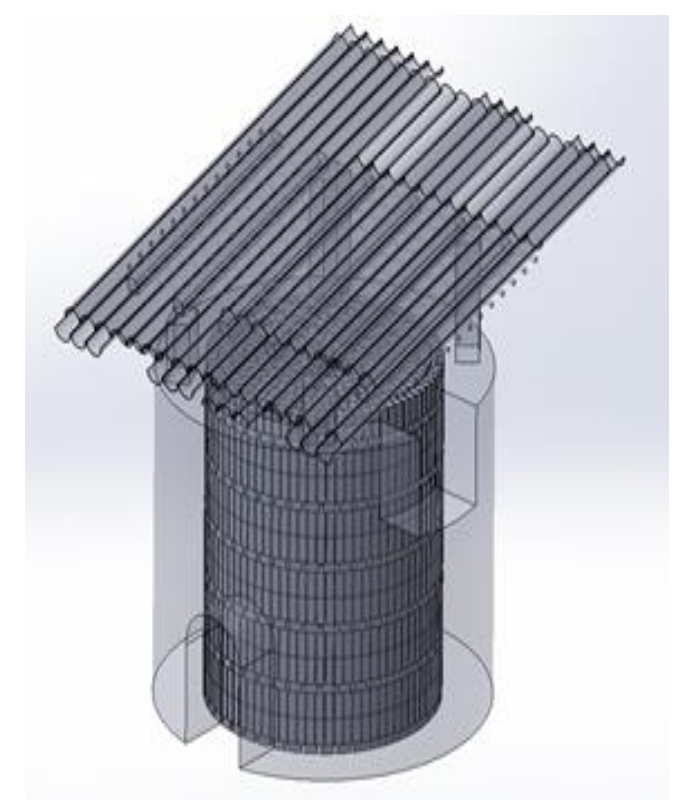

Fig. 1. Cargue del ladrillo y combustible en horno artesanal a cielo abierto

El proceso de cocción inicia cuando se empieza a inyectar aire para la combustión al hogar o parrilla del horno por medio de un ventilador, empezando la cocción en la parte inferior del horno y ascendiendo a medida que se va consumiendo el carbón, (Ver figura 2), hasta que se queme todo el carbón mineral. Luego se abre la puerta del horno y se deja un periodo de enfriamiento para poder sacar el ladrillo. 


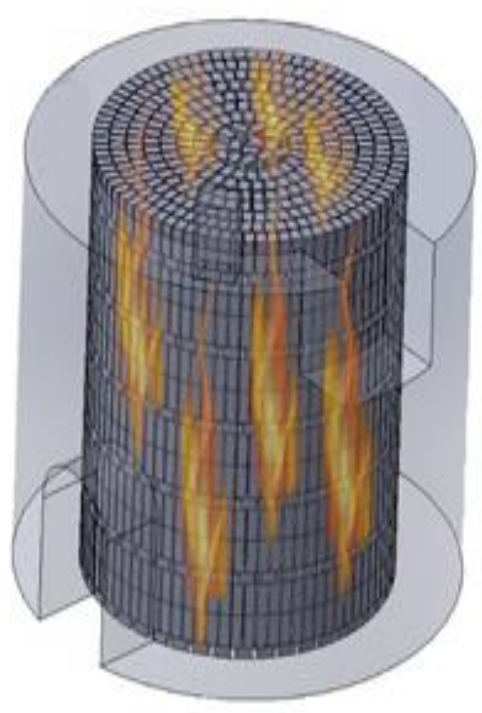

\section{Fig. 2. Proceso de cocción en horno artesanal a} cielo abierto

Luego se procedió a seleccionar 20 ladrillos cocidos en diferentes posiciones en el horno para la evaluación de las propiedades mecánicas de los ladrillos macizos por medio de pruebas estandarizadas de control de calidad no destructivas y destructivas de los ladrillos de mampostería utilizando la norma técnica Colombina NTC-4017 "Métodos para muestreo y ensayos de unidades de mampostería y otros productos de arcilla". Las pruebas o ensayos que se utilizaron fueron tasa de absorción inicial, ensayo de inmersión durante 24 horas, resistencia a la compresión y módulo de rotura o flexión y en cada ensayo se seleccionaron 5 muestras de ladrillos. Para los ensayos de resistencia a la compresión y módulo de rotura se utilizó una máquina Universal de ensayos tipo Pinzuar PU-100 con capacidad de 1000 kN y el software estadístico Statgraphics.

En la segunda parte de la investigación se seleccionaron 20 muestras crudas de ladrillos que se hicieron en la ladrillera la Pradera y se llevaron al horno continuo tipo Hoffman de la ladrillera Ocaña, el proceso de cargue de estas muestras se hizo a medida que se iba cargando un apile con bloques H 10 en el horno Hoffman, (Ver figura 3). Luego se realizó el proceso de cocción, la cual tuvo una duración de un día y se extrajeron las muestras macizas a las que se les determinaron las propiedades mecánicas.

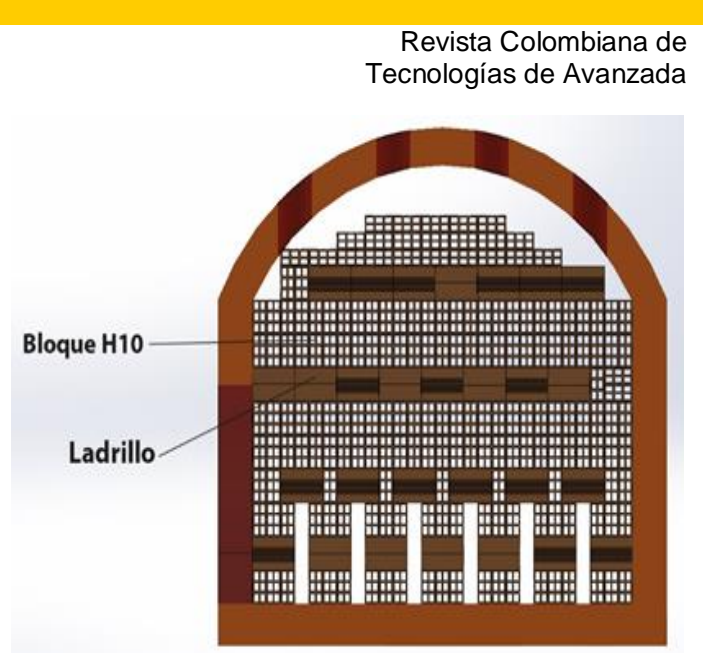

Fig. 3. Cargue de bloques H 10 y ladrillo en horno continuo

Finalmente se determinó la tasa de absorción inicial e inmersión durante 24 horas, resistencia a la compresión y a la flexión, que permitieron identificar las características y propiedades de los materiales que conforman los ladrillos, estableciendo una comparación con los parámetros de absorción, resistencia a la compresión y flexión entre las muestras de los dos hornos y con los parámetros establecidos en la norma NTC 4205 unidades de mampostería de arcilla cocida, ladrillos y bloques cerámicos.

La ladrillera Ocaña está dedicada a la producción en serie de unidades de perforación horizontal $\mathrm{H}$ 10 para la construcción, dicho proceso de producción es mecanizado. El horno está compuesto de 24 cámaras o puertas (12 en cada lado). En cada puerta se introducen 5 apiles, de 620 bloques aproximadamente, separados $60 \mathrm{~cm}$ entre ellos (Ver figura 4).

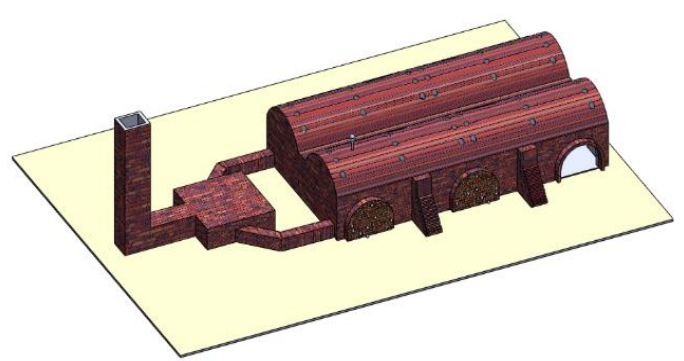

Fig. 4. Horno Hoffman

El combustible utilizado para la cocción del material es carbón pulverizado, con un consumo de carbón por mes es de 170 Ton/ mes (Marrugo, 2015), el cual es inyectado en los agujeros ubicados en la parte superior del horno por medio de dos carbojet a razón de $8 \mathrm{~kg}$ a $12 \mathrm{~kg}$ de carbón 
por minuto, donde uno de estos va primero precalentando dos cámaras antes de que pase el segundo carbojet requemando el ladrillo, (Ver figura 5).

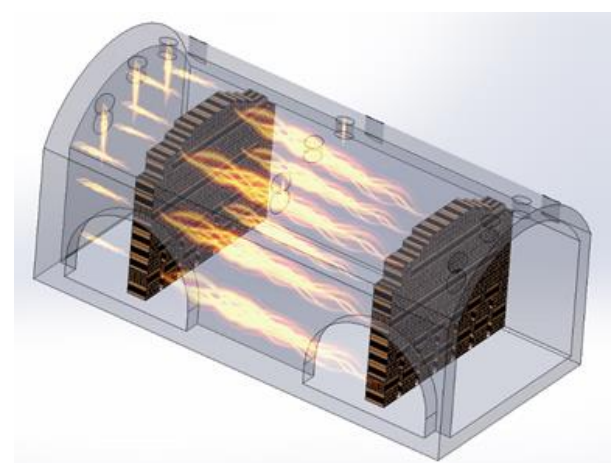

Fig. 5. Proceso de cocción en horno continúo

\subsection{Ensayos}

\subsubsection{Ensayo tasa de absorción inicial}

El ensayo mide la cantidad de agua que absorbe el ladrillo en 1 minuto. El ensayo consistió en determinar el área de la superficie de la muestra en contacto con el agua, la masa seca de la muestra y la masa final de la muestra, (Ver figura 6). Para medir las muestras se utilizó un calibrador digital marca standardgage de $350 \mathrm{~mm}$ con tolerancias de $0.1 \mu \mathrm{m}$ y para pesarlos una balanza electrónica digital marca pinzuar modelo PG89.

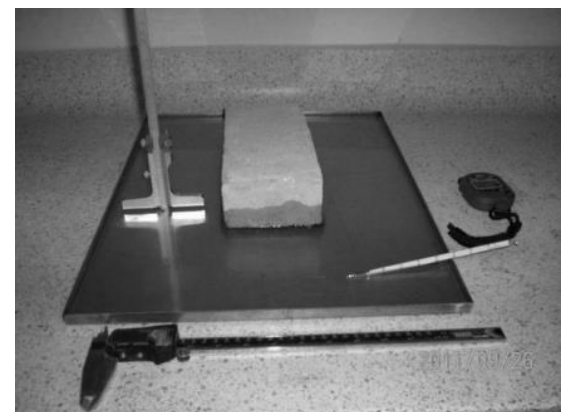

Fig. 6. Ensayo tasa de absorción inicial en unidades de mampostería

Para la evaluación de la tasa de absorción inicial en las muestras se utilizó la siguiente ecuación:

$$
\mathrm{TLA}=\frac{\mathrm{G}}{\mathrm{A}}
$$

Donde T.I.A. es la tasa de absorción inicial dada en $\left(\mathrm{g} / \mathrm{cm}^{\wedge} 2 / \mathrm{mín}\right.$.), $\mathrm{G}$ es la diferencia entre la masa final e inicial de la muestra por cada minuto dada en (g/min.) y A es el área de la muestra en contacto con el agua dada en $\left(\mathrm{cm}^{\wedge} 2\right)$.

\subsubsection{Ensayo Inmersión durante 24 horas}

El ensayo mide la cantidad de agua que absorbe el ladrillo durante 24 horas, para la realización del ensayo las muestras se secaron a una temperatura de $110{ }^{\circ} \mathrm{C}$ durante 24 horas en un horno mufla marca Pinzuar, luego se colocaron en una cámara de enfriamiento a una temperatura de $24{ }^{\circ} \mathrm{C} \pm 8^{\circ} \mathrm{C}$ y humedad entre el $30 \%$ y $70 \%$ durante 4 horas; en ese instante se determinó la masa seca de cada muestra, luego se sumergieron en un tanque con agua a una temperatura de entre $15{ }^{\circ} \mathrm{C}$ y $30{ }^{\circ} \mathrm{C}$ durante 24 horas, las muestras se sacaron del tanque y se procedió a determinar la masa final de la muestra después del ensayo.

Para la evaluación de la absorción durante las 24 horas en las muestras se utilizó la siguiente ecuación:

$$
\text { A.F. }=((\mathrm{mss}-\mathrm{ms})) / \mathrm{ms} * 100
$$

Donde A.F. es la absorción de agua en la muestra durante 24 horas dada en $\%$, mss es la masa final de la muestra después del ensayo dada en ( $\mathrm{g}$ ) y ms es la masa seca de la muestra dada en $(\mathrm{g})$.

\subsubsection{Ensayo resistencia a la compresión}

El ensayo consistió en llevar la muestra a la falla para determinar el esfuerzo máximo de compresión (Takeuchi ,2007). El procedimiento para el secado de las muestras fue el mismo que se les realizó a las muestras en el ensayo de absorción de agua; luego a las muestras se les aplicó una capa delgada de 3 milímetros de yeso a las caras opuestas a la aplicación de la carga, finalmente se llevaron las muestras a una máquina universal, (Ver figura 7).

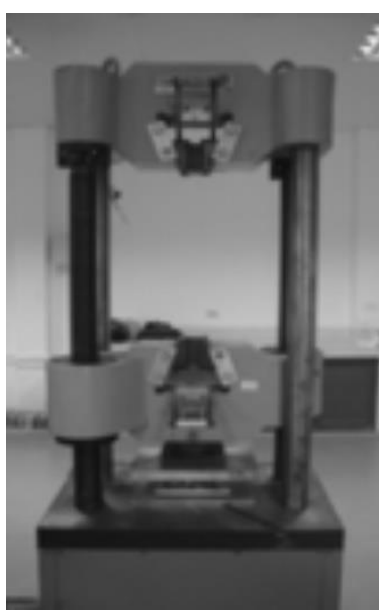

Fig. 7. Ensayo resistencia a la compresión 
Para la evaluación de la resistencia a la compresión en las muestras se utilizó la siguiente ecuación:

$$
\mathrm{f}_{\mathrm{cu}}=\frac{\mathrm{W}}{\mathrm{A}}
$$

Dónde $\mathrm{f}_{\mathrm{cp}}$ es la resistencia de la muestra a la compresión dada en (MPa), W es la carga máxima de rotura dada en $(\mathrm{N})$ y A es el área promedio de las superficies superior e inferior de la muestra dada en $\left(\mathrm{mm}^{\wedge} 2\right)$.

\subsubsection{Ensayo módulo de rotura o flexión}

El ensayo consistió en llevar la muestra a la falla para determinar el módulo de flexión (Kaushik, 2007). La preparación de las muestras en el ensayo de rotura es la misma que en el ensayo de compresión, en este ensayo la muestra se coloca entre dos apoyos y es sometida a una carga puntual en el centro de la luz de la cara superior de la muestra mediante una placa de acero (Ver figura 8).

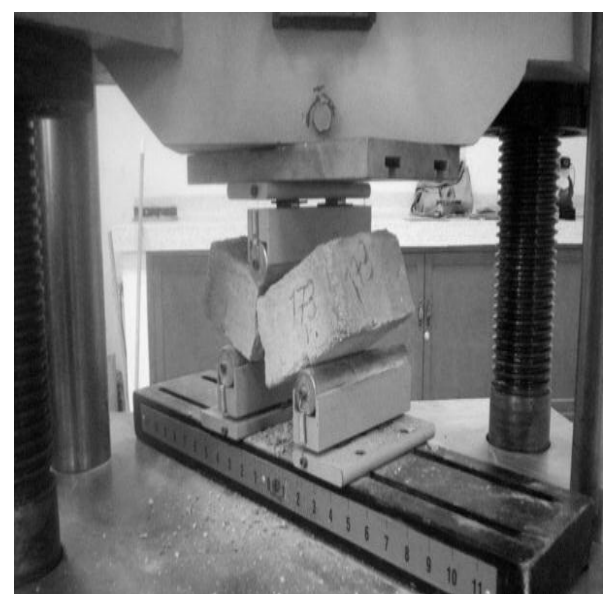

Fig. 8. Ensayo módulo de rotura o flexión

Para la evaluación de módulo de rotura en las muestras se utilizó la siguiente ecuación:

$$
M R=\frac{a W \cdot\left(\frac{L}{2}-x\right)}{b \cdot d^{2}}
$$

Donde MR es el módulo de rotura de la muestra dada en (MPa), W es la carga máxima de rotura dada en (N), L es la distancia entre los apoyos dada en $(\mathrm{mm}), \mathrm{b}$ es el ancho de la muestra en el plano de falla dada en $(\mathrm{mm})$, d es la profundidad de la muestra en el plano de falla dada en $(\mathrm{mm})$ y X es la distancia promedio del plano de falla al centro de la pieza, medida en la dirección de la línea central de la superficie sometida a tensión dada en $(\mathrm{mm})$.

\section{RESULTADOS}

\subsection{Análisis de ensayos}

El resultado de los ensayos tasa de absorción inicial e inmersión durante 24 horas de las muestras entre los hornos a fuego dormido y continuo respecto a la norma pueden verse en las figuras 9 y 10.

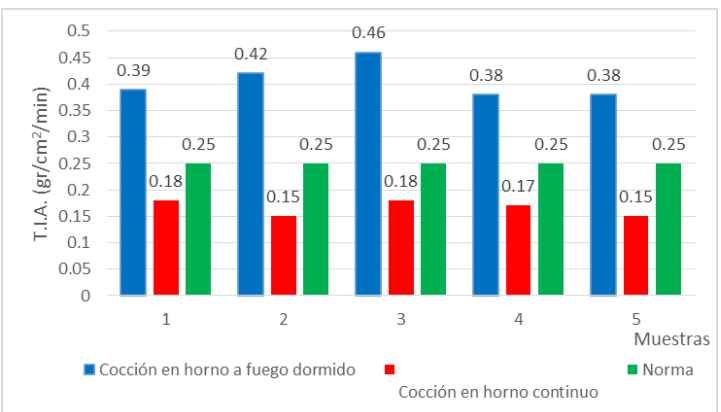

Fig. 9. Comparación resultados ensayo tasa de absorción inicial entre hornos y norma.

La tasa de absorción inicial de agua en las muestras del horno a fuego dormido tiene una variación que oscila entre $0.38 \mathrm{~g} / \mathrm{cm}^{\wedge} 2 / \mathrm{mín}$ y $0.46 \mathrm{~g} / \mathrm{cm}^{\wedge} 2 / \mathrm{mín}$, con un promedio de $0.41 \mathrm{~g} / \mathrm{cm}^{\wedge} 2 / \mathrm{mín}$; mientras que en las muestras del horno continuo tiene una variación que oscila entre $0.15 \mathrm{~g} / \mathrm{cm}^{\wedge} 2 /$ mín. y 0.18 $\mathrm{g} / \mathrm{cm}^{\wedge} 2 / \mathrm{mín}$., con un promedio de 0.17 $\mathrm{g} / \mathrm{cm}^{\wedge} 2 / \mathrm{mín}$.

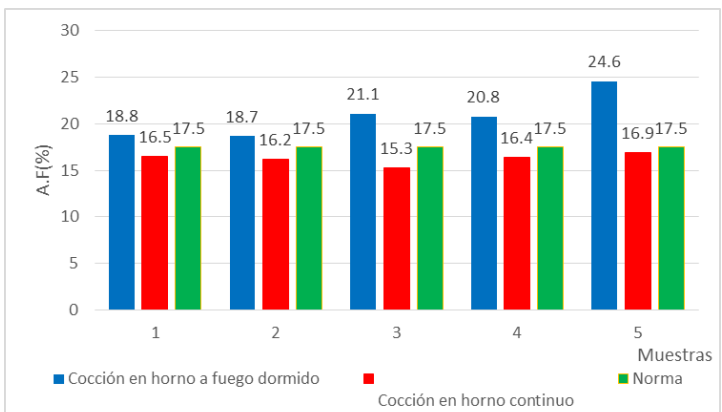

Fig. 10. Comparación resultados ensayo absorción 24 horas entre hornos y norma.

La absorción de agua durante 24 horas en las muestras del horno a fuego dormido tiene una variación que oscila entre $18.7 \%$ y $24.6 \%$, con un promedio de $20.8 \%$; mientras que en las muestras del horno continuo tiene una variación que oscila entre $15.3 \%$ y $16.9 \%$, con un promedio de $16.3 \%$.

Los resultados obtenidos en el ensayo resistencia a la compresión en las muestras de los hornos a 
fuego dormido y continuo se muestra en la figura 11.

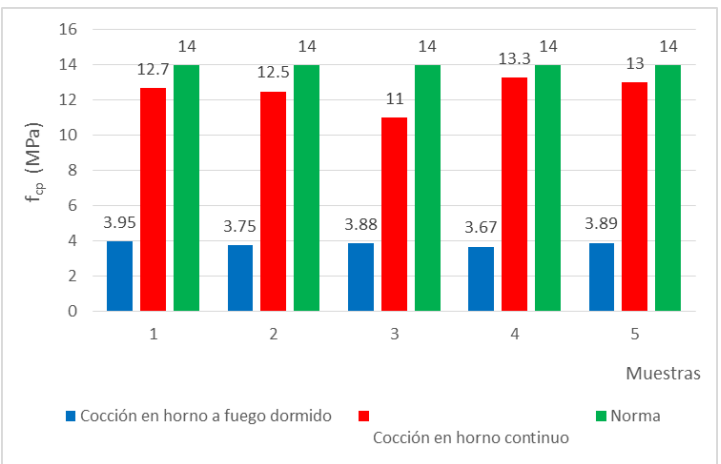

Fig. 11. Resultados ensayo resistencia a la compresión.

La resistencia a la compresión en las muestras del horno a fuego dormido tiene una variación que oscila entre $3.67 \mathrm{MPa}$ y $3.95 \mathrm{MPa}$, con un promedio de $3.83 \mathrm{MPa}$; mientras que en las muestras del horno continuo tiene una variación que oscila entre $11 \mathrm{MPa}$ y $13.3 \mathrm{MPa}$, con un promedio de $12.5 \mathrm{MPa}$.

Los resultados obtenidos en el ensayo módulo de rotura en las muestras de los hornos a fuego dormido y continuo se muestra en la figura 12.

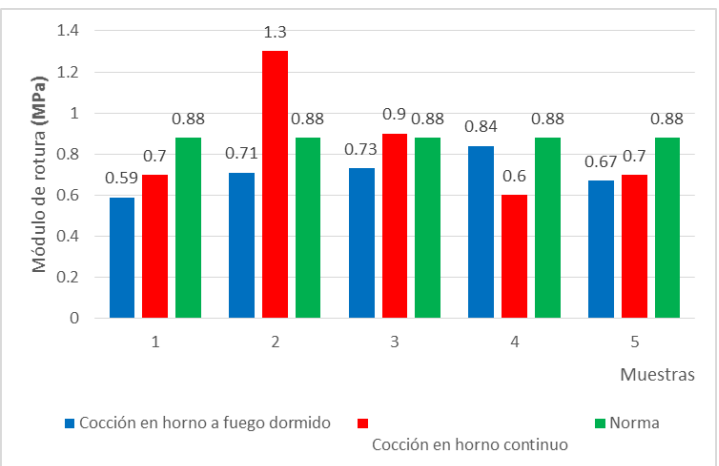

Fig. 12. Resultados ensayo módulo de rotura

El módulo de rotura en las muestras del horno a fuego dormido tiene una variación que oscila entre $0.59 \mathrm{MPa}$ y $0.84 \mathrm{MPa}$, con un promedio de 0.71 $\mathrm{MPa}$; mientras que en las muestras del horno continuo tiene una variación que oscila entre 0.6 MPa y 1.3 MPa, con un promedio de $0.84 \mathrm{MPa}$.

\section{CONCLUSIONES}

El valor promedio en la tasa de absorción inicial de agua en las muestras del horno a fuego dormido es de $0.41 \mathrm{~g} / \mathrm{cm}^{\wedge} 2 / \mathrm{mín}$., mientras que en las muestras del horno continuo es de $0.17 \mathrm{~g} / \mathrm{cm}^{\wedge} 2 / \mathrm{mín}$, representando $141.18 \%$ más de absorción de agua por minuto en las muestras del horno fuego dormido respecto al horno continuo.

Las muestras del horno continuo cumplen con lo establecido en la norma NTC 4205 que establece un valor de $0.25 \mathrm{~g} / \mathrm{cm}^{\wedge} 2 / \mathrm{mín}$; mientras que las muestras del horno a fuego dormido no cumplen con lo establecido en la norma, el valor de absorción inicial en las muestras del horno a fuego dormido es $64 \%$ más que el valor que establece la norma. Estos altos valores podrían deberse a una mezcla deficiente, mal proceso de trituración artesanal, deficiente proceso de cocción por parte del fabricante, lo que genera presencia de poros superficiales permitiendo que el ladrillo absorba gran cantidad de agua rápidamente afectando la adherencia y consistencia de mortero.

El valor promedio en la absorción de agua durante 24 horas en las muestras del horno a fuego dormido es de $20.8 \%$, mientras que en las muestras del horno continuo es de $16.3 \%$, representando $27.6 \%$ más de absorción de agua por minuto en las muestras del horno a fuego dormido respecto al continuo.

Las muestras del horno continuo cumplen con lo establecido en la norma NTC 4205 que establece un valor de $17.5 \%$; mientras que las muestras del horno a fuego dormido no cumplen con lo establecido en la norma, el valor de absorción 24 horas en las muestras del horno a fuego dormido es 18.9 \% más que el valor que establece la norma.

El valor promedio en la resistencia a la compresión en las muestras del horno a fuego dormido es de 3.83 MPa; mientras que en las muestras del horno continuo es de $12.5 \mathrm{MPa}$, representando $226.4 \%$ más en la resistencia a la compresión en las muestras del horno continuo respecto a las de fuego dormido.

Ninguna de las dos muestras cumple con lo establecido en la norma NTC 4205 que establece un valor de $14 \mathrm{MPa}$; el valor en la resistencia a la compresión en las muestras del horno a fuego dormido es $265.5 \%$ menos que el valor que establece la norma y $12 \%$ menos en los del horno continuo.

El valor promedio en el módulo de rotura en las muestras del horno a fuego dormido es de 0.71 $\mathrm{MPa}$; mientras que en las muestras del horno continuo es de $0.84 \mathrm{MPa}$, representando $18.3 \%$ 
más en la resistencia a la flexión en las muestras del horno continuo respecto a las de fuego dormido.

Ninguna de las dos muestras cumple con lo establecido en la norma NTC 4205 que establece un valor de $0.88 \mathrm{MPa}$; el valor en la resistencia a la flexión en las muestras del horno a fuego dormido es $23.94 \%$ menos que el valor que establece la norma y $4.8 \%$ menos en los del horno continuo.

Los valores de las propiedades en las muestras más cercano a la norma fue la cocción que se hizo en el horno continúo debido a la tecnología de horno empleado para la cocción, por tal motivo se recomienda hacer uso de hornos con buena eficiencia energética que permitan disminuir el consumo del combustible, reduzca los tiempos de cocción, reutilicen los gases de escape mejorando la combustión del carbón y disminuyan las emisiones emitidas a la atmosfera. Otro aspecto en la adecuación en los hornos es la construcción de la chimenea la cual mejoraría el tiro en el horno y facilitará el oxígeno para que la combustión sea completa. Además de la preparación y calidad del combustible hasta el cambio de combustible a gas natural, ya que el horno intercambia calor por radiación entre la superficie interior con la carga, y por convección entre el fluido y las superficies de pared y de carga.

\section{REFERENCIAS}

Guerrero,G. (2017). Hornos de combustión: alternativas de reducción de emisiones e incremento de rentabilidad. Universidad de Santander Bucaramanga.

Garcia,J. (2019). Energetic analysis in traditional panelers burners. IOP Conf. Series: Journal of Physics: Conf. Series 1257 (2019) 012017. IOP Publishing. doi:10.1088/17426596/1257/1/012017

Guerrero,G. (2017). Thermodynamic balance: Continuous oven. IOP Conf. Series: Journal of Physics: Conf. Series 1126 (2018) 012025 doi :10.1088/1742-6596/1126/1/012025

Guerrero,G. (2017). Análisis isocinético y corrección a condiciones de referencia en horno a cielo abierto en el municipio de Ocaña, Norte de Santander. REVISTA INGENIO UFPSO - Vol. 14 - Enero-Junio
2017 - p-ISSN 2011-642X e-ISSN 2389-

$864 X$ - Edición Especial

Rincón C., Gil J., Lesmes C., Caro C. (2016). Evaluación de la sostenibilidad de la producción de ladrillo en la región de Boyacá, Colombia. Revista L'esprit Ingéniux e-ISSN: 2422-2445, Enero-Diciembre 2016, No. 3545.

Rozo, S., \& Sánchez, J. A. (2014). Propiedades físico mecánicas de bloques H10 fabricados en el área metropolitana de Cúcuta. 24(1).

Aranguren, A., \& Sancho, J. (2011). Método de Caracterización de las Propiedades mecánicas de la Fractura del Ladrillo. 2.

Asociación de Ingeniería Sísmica, (AIS). (2004). "Guía de Patologías constructivas, estructurales y no estructurales”. Santafé de Bogotá D. C. Colombia.

Cuellar E., Portillo J., Renderos M., y Vides F., (2006). Evaluación de la resistencia a la fractura de los ladrillos de barro fabricados por compresión. Trabajo de grado no publicada, Universidad Centroamericana “José Simeón Cañas”. San Salvador.

Francis A., Rawlings R., Sweeney R., Boccaccini A. (2002) Processing of coal ash into glass ceramic products by powder technology and sintering, Glass Technol, 43: 58-62.

Barbieri L., Lancelloti I., Manfredini T., Queralt I., Rincón J., Romero M. (1999) Glasses and glass-ceramics from coal fly ashes. Fuel 78: 271-276.

Souza M. F., Yamamoto J. (1999) Mullite synthesised from aluminium hydroxide and silica from rice hull, Cerâmica, 45: 34-38.

Skarkynska K. (1995) Engineering- part 1: properties of mine stone; reuse of coal mining wastes in civil, Waste Management, 15: 3-42.

Instituto Colombiano De Normas Técnicas NTC, «Métodos para Muestreo y Ensayos de Unidades de Mampostería y Otros Productos de Arcilla NTC4017),» Bogotá D.C., 2005.

Instituto Colombiano De Normas Técnicas NTC (2000). Unidades de Mampostería de Arcilla 
Cocida. ladrillos y bloques crámicos NTC 4205. Bogota D.C.,

JEG Plaza, MAR Núñez, (2017) Formación en competencias específicas para la industria del software colombiano. Experiencias del uso del aprendizaje basado en proyectos. Revista Colombiana de Tecnologías de Avanzada, ISSN: 1692-7257.

L Tangarife, M Sánchez, M Rojas (2017). Modelo de interventoría de tecnologías de información en el área de conocimiento de la gestión del alcance de PMBOK® y alineado con ISO 21500 y COBIT®. Revista Colombiana de Tecnologías de Avanzada, ISSN: 1692-7257.

Marrugo D, (2015). Desarrollo de un instrumento virtual para la generación de perfiles de temperatura en hornos. Universidad Francisco de Paula Santander Ocaña. Ingeniería Mecánica. 2015.
O Suarez, C Vega, E Sánchez, A Pardo. (2018) Degradación anormal de p53 e inducción de apoptosis en la red P53-mdm2 usando la estrategia de control tipo pin. Revista Colombiana de Tecnologías de Avanzada, ISSN: 1692-7257.

Takeuchi C, (2007). Comportamiento en la mampostería estructural, Universidad Nacional de Colombia, Santa fe de Bogotá.

Kaushik H, Rai D. y Jain S., (2007). "Stress-strain characteristics of clay brick masonry under uniaxial compression", Journal of materials in civil engineering ASCE, September, pg. 728 -739 . 